\title{
User-Friendly Software for the Analysis of Complex XRF Spectra
}

\author{
Lisa Van Loon, Stewart McIntyre, Nathaniel Sherry, Michael Bauer and Neil Banerjee \\ Western University, London, Ontario, Canada
}

\section{Introduction}

Peakaboo is an XRF spectral analysis platform that is freely accessible to users from all scientific communities [1-3]. It accepts a wide range of data formats for both synchrotron and conventional sources. The software guides users through peak assignments, quantitative analysis, and 2D mapping for large, full-spectrum data sets. The quick learning curve means that new users can become comfortable with the main program functions in a single session. Designed for both expert and new users from all fields, the spectral fitting is designed to avoid common fitting mistakes. Complex spectra of overlapping peaks are resolved using details of the shapes of individual K, L and M peaks. Anomalous sources of peaks such as summation processes, diffraction and escape peaks are readily identified, thus avoiding errors in peak assignment. Estimates of elemental concentrations are made using reference materials and measurement protocols that can be applied within minutes. Large data sets can be rapidly processed and displayed as 2D element maps and correlation plots.

\section{Accurate Fitting of Overlapping Spectral Peaks}

Figure 1a shows the main page containing a cleanly-defined set of icons that provide three different methods for elemental identification:(a) click on the peak itself to receive a list of suggestions, (b) request an auto spectrum analysis, or (c) select the peak manually from a provided table. Identification of all possible peak summation processes is available (Figure 1b). Characteristic K, L and M peaks are matched against spectral data collected by the xraylib project [4]. K, L and M peak shapes and separations and relative intensities are unique for each element. Thus, to make a positive element identification, users can overlay an unknown shape with a representation of the expected spectrum for that element. This also provides a much more certain measure of peak intensity than the "ROI" measurement that is still in common practice. The peak overlay approach allows new users to visually observe and understand the contributions of each element to the full, collected XRF spectrum, resulting in a more sophisticated analysis.

Multi-element spectra always contain many overlapping peaks; in Peakaboo these are managed with a rapid and exacting peak fitting routine. Overlapping peaks are resolved through a very fast fitting routine (2-3 seconds) that minimizes errors between the actual spectrum and the fitted peak shapes. Several separate fitting sequences are averaged, each one beginning in a different part of the spectrum. A pseudoVoigt peak shape is used and peak width is set by the Fano noise convention. An example of overlapping $\mathrm{K}$ and $\mathrm{L}$ peaks fitted and identified is shown in Figure 1c. An important observation is that even peaks with low intensities, such as many of the rare earth elements, can be identified in the presence of the more intense $\mathrm{K}$ peaks from the first and second rows of the periodic table.

\section{Estimation of Concentrations}

With a substantial increase in the number of elements detected, the next stage has been to provide an estimation of their relative concentrations. To assess concentrations in low $\mathrm{Z}$ chemical matrices the NIST SRM 610 was used as a reference standard that is measured alongside an unknown material. The resultant 
spectrum is then fitted automatically and a table of sensitivity coefficients is generated from the known concentrations. As a test, the element concentrations for NIST SRM 614 were determined within a factor of two of their reference values. This accuracy is sufficient to allow the software to rapidly make a firstpass, quantitative analysis.

\section{Advances in Spectral Mapping and Correlation}

In recent years, XRF spatial maps have increased in size as fly-scanning technology has become available. Peakaboo is capable of rapidly processing both single and multi-element 2D maps from the analyzed, fullspectrum XRF data. The software can easily create maps containing tens of thousands of pixels from detailed micron-scale maps to cm-scale maps. Its mathematical filtration tools are particularly important for improvement of low granularity images. The mapped intensities can be scaled to the visible fittings or to all fittings. A series of single-element maps showing the compositional distribution of several elements in a uranium ore sample is shown in Figure 2a. Further interrogation in the software shows that the distribution of uranium by itself is markedly different from the uranium that is associated with arsenic (Fig 2b). These relationships can also be presented as correlation plots created in the software (not shown here).

\section{Conclusion}

The Peakaboo analysis platform is evolving rapidly to support the evolving needs of its users. New capabilities, particularly in the areas of quantification and more elaborate image analysis are continually being developed.

\section{Acknowledgements}

Part of the research described in this paper was performed at the Canadian Light Source, a national research facility of the University of Saskatchewan, which is supported by the Canada Foundation for Innovation (CFI), the Natural Sciences and Engineering Research Council (NSERC), the National Research Council (NRC), the Canadian Institutes of Health Research (CIHR), the Government of Saskatchewan, and the University of Saskatchewan. This research also used resources of the Advanced Photon Source, an Office of Science User Facility operated for the U.S. Department of Energy (DOE) Office of Science by Argonne National Laboratory, and was supported by the U.S. DOE under Contract No. DE-AC02-06CH11357, the Canadian Light Source and its funding partners. We thank R. Feng, Z. Finfrock, and D. Motta Meira for support during data collection. We thank Sigray Inc. and W. Yun and B. Stripe for support in collecting data. The authors acknowledge funding from CANARIE and NSERC. 


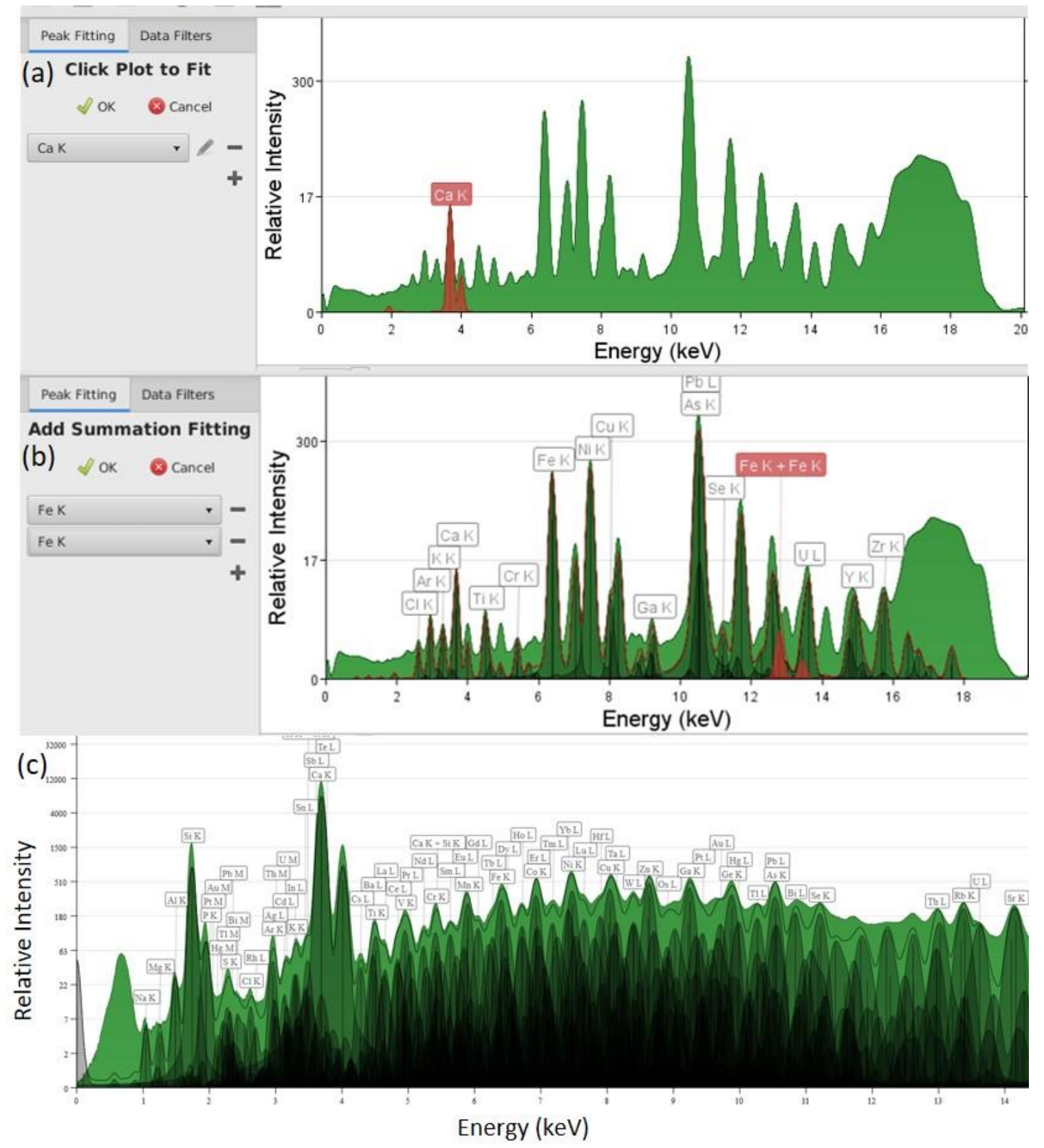

Figure 1. (a). Elemental Identification by Clicking on a Peak. The contributions from the $\mathrm{Ca} \mathrm{Ka}$ and $\mathrm{K} \beta$ peaks are shown in red at $\sim 4 \mathrm{keV}$. The summation peaks are shown in red at $\sim 2 \mathrm{keV}$. (b) Automated Summation Peak Fitting. The Fe K + Fe K summation peak contributions are shown in red at $\sim 13 \mathrm{keV}$. The Fe Ka and $\mathrm{Kb}$ peaks are observed at $~ 6.4$ and $7 \mathrm{keV}$. (c) XRF Spectrum of NIST glass SRM 610 collected on a Sigray, Inc. prototype Attomap XRF spectrometer using Mo Ka radiation. Collection time was 10 minutes. Peak noise was 70 mv. Spectral peaks from K, L and M lines are shown, along with loss peaks and some summation peaks. The solid line represents the summation of all peak intensities; as such it is measure of the goodness of the fit. At higher energies, the somewhat lesser goodness of fit is probably due to an accumulation of many summation peaks. 

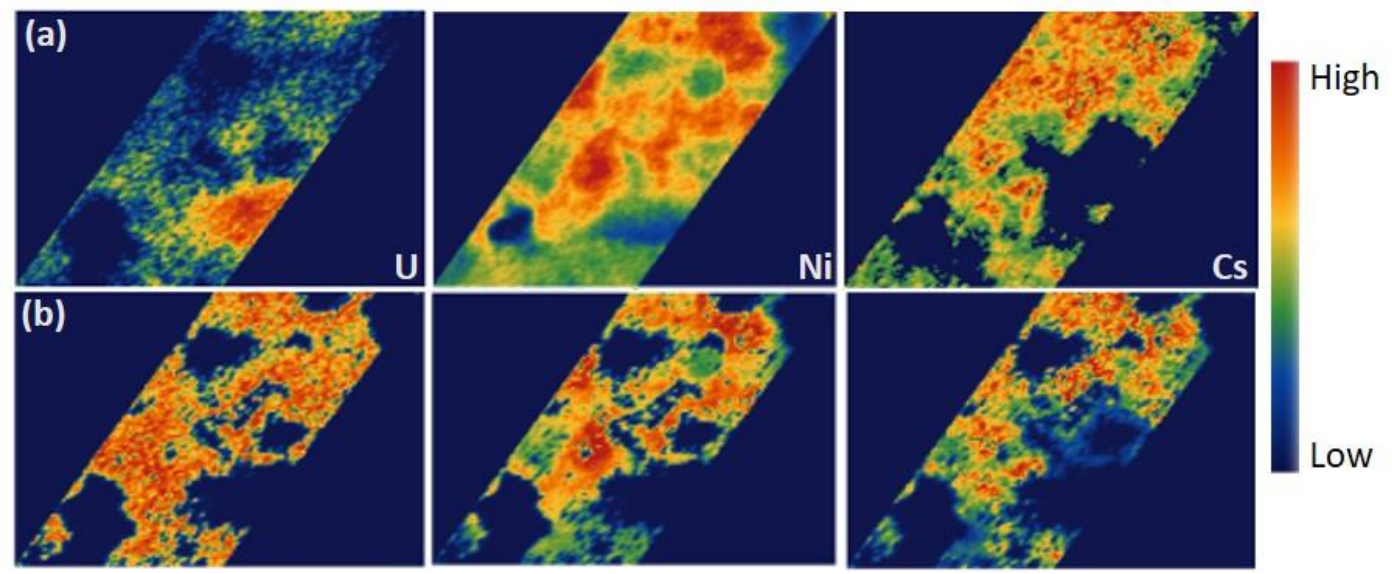

Figure 2. (a). Individual 2D XRF element maps of U, Ni, and Cs. The relative intensities for each map are represented using a colour scale from red (highest) to blue (lowest). (b) Correlation maps where the distribution of $\mathrm{U}, \mathrm{Ni}$, and $\mathrm{Cs}$ have been correlated with the distribution of As. The data was collected at the VESPERS beamline at the CLS.

\section{References}

[1] Van Loon, L. L., McIntyre, N. S., Bauer, M., Sherry, N. S. A. \& Banerjee, N. R. (2019). Software Impacts. 2, 100010.

[2] http://www.peakaboo.org

[3] N. Sherry, J. Qin, M. Suominen-Fuller, Y. Xie, O. Mola, M. Bauer, N.S. McIntyre, D. Maxwell, D. Liu, E. Matias and C. Armstrong, Analytical Chemistry, 2012, 84, 7283-7291. 